\title{
Shedding light on a hidden source of septic shock with POCUS
}

\author{
Miguel Lourenço Varela, MD¹; Rita Martins Fernandes, $\mathrm{MD}^{2}$; Maria Luísa Melão, MD¹; Javier Moreno, MD ${ }^{1}$; Cristina \\ Granja, MD, PhD ${ }^{1,3,4}$ \\ (1) Intensive Care Medicine 1, Hospital de Faro, Centro Hospitalar Universitário do Algarve \\ (2) Internal Medicine 3, Hospital de Faro, Centro Hospitalar Universitário do Algarve \\ (3) CINTESIS - Center for Health Technology and Services Research, Faculty of Medicine, University of Porto, Porto, Portugal
}

(4) Department of Biomedical Sciences and Medicine, University of Algarve, Faro, Portugal

\begin{abstract}
A 77-year old male was admitted in the emergency department for septic shock, yet no clear source of infection was noted upon physical examination and a portable chest x-ray. Due to his unstable condition, bedside ultrasound was performed. A heterogeneous mass in the liver was noted, hence a tentative diagnosis of liver abscess was made. This was latter confirmed by abdominal computed tomography. This case highlights that point-of-care ultrasound, when performed by expert physicians, can significantly decrease time to diagnosis for septic patients.
\end{abstract}

\section{Case file}

A 77-year old male presented to our hospital's emergency department complaining of acute shortness of breath and 1 week of malaise. He complained of nausea and vomiting in that period, without abdominal pain, diarrhea, cough, chest pain or dysuria. His past history included acute pulmonary thromboembolism 15 years prior and dyslipidemia.

Upon examination, he had a Glasgow Coma Scale of 15, was shivering, hypotensive (mean arterial blood pressure of $40 \mathrm{mmHg}$ ), tachycardic (140 beats per minute), hypoxic (peripheral oxygen saturation of $82 \%$ on room air) and febrile (temperature of $38.9^{\circ} \mathrm{C}$ ). An arterial blood gas on $6 \mathrm{~L} / \mathrm{min}$ of oxygen through a simple face mask revealed a compensated acute metabolic acidosis, with a $\mathrm{pH}$ of 7.404, pCO2 $15 \mathrm{mmHg}$, pO2 $101 \mathrm{mmHg}$, HCO3 15.4 $\mathrm{mmol} / \mathrm{L}$ and lactate of $10.4 \mathrm{mmol} / \mathrm{L}$. He was admitted to the Emergency Room's Critical Care bay upon suspicion of septic shock. Sepsis protocols were immediately started and upon further examination, there still was no obvious source of infection: he had a clear lung exam, there was no abdominal or costovertebral angle tenderness and he had a normal prostate exam.

The chest $\mathrm{x}$-ray had no visible consolidation. The patient's blood pressure improved only transiently after fluid therapy; because the patient continued to be unstable, a bedside ultrasound examination was performed to further look for a source of infection. It revealed a heterogeneous mass in the liver (Figure 1A). A liver abscess was suspected. An abdominal computed tomography scan was then performed, which revealed the aforementioned abscess located in the $\mathrm{VI}$ and $\mathrm{VII}$ liver segments, measuring $13 \times 7 \times 10 \mathrm{~cm}$ (Figure 1B) and signs of a contained perforated diverticulitis.
Metronidazole and gentamicin were added to ceftriaxone that had already been administered. Percutaneous drainage revealed a thick purulent material; cultures later isolated an extended-spectrum beta-lactamase producer, Morganella morganii.

The patient's status rapidly deteriorated and he was started on vasopressor therapy, intubated and admitted to the Intensive Care Unit (ICU). Abscess drainage and appropriate antibiotic therapy led to steady improvement, both clinically and on repeat imaging exams (Figure 1C). $\mathrm{He}$ was later transferred to the intermediate care unit continuing on antibiotic therapy.

Septic shock has a high mortality rate, estimated to be higher than $40 \%$ [1], increasing as time passes without appropriate treatment. Hence, correct diagnosis of the cause of sepsis is essential to provide correct antibiotic coverage and, if necessary, drainage or removal of the infectious source. However, patients with septic shock are, by definition, hemodynamically unstable [1], hence it is reasonable that attempts at transportation of these patients for diagnostic tests should be keep at a minimum.

Pyogenic liver abscess is an uncommon entity in Western countries, yet clearly shows the need of quickly pinpointing the source of a septic shock, as it historically carried a high mortality rate, that is currently at $6.3 \%$ due to the generalized use of computed tomography and ultrasound [2]. There is usually a delay of 1 week between onset of symptoms and diagnosis, as most of them are nonspecific and there are multiple occult sources of infection, such as diverticulitis [3].

Point-of-care ultrasound, when performed by expert physicians, significantly decreases time to diagnosis of septic patients [4], potentially decreasing time to control 

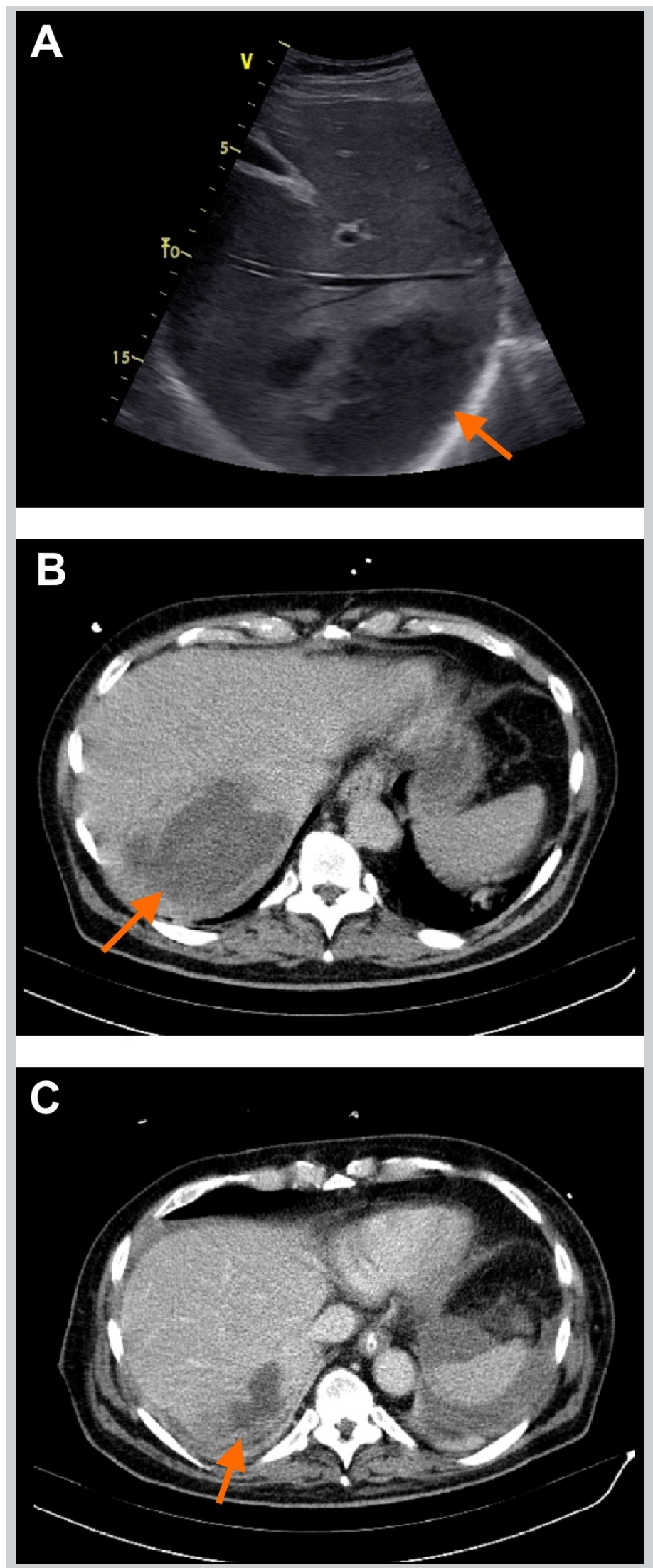

Figure 1. Abdominal point-of-care ultrasound showing an heterogeneous liver lesion ( $1 \mathrm{~A}$, arrow), confirmed as a liver abscess in the contrast-enhanced abdominal computed tomography (1B, arrow). There was significant decrease of the abscess after two weeks of therapy (1C, arrow). of the septic source, while maintaining the patient in a more controlled environment and arranging for further testing. Also a quick identification of the septic source may help eliminate sepsis mimickers that could affect the differential diagnosis process. These advantages could be of benefit for patient outcomes, although, interestingly, patients with septic shock admitted to the ICU without a clear diagnosis at $24 \mathrm{~h}$ had no difference in outcomes [5]. Studies to correlate use of point-of-care ultrasound in these patients with benefits in mortality are needed.

\section{References}

1. Singer M, Deutschman C, Seymour C, Shankar-Hari M, Annane D, Bauer $M$ et al. The Third International Consensus Definitions for Sepsis and Septic Shock (Sepsis-3). JAMA. 2016;315(8):801. DOI: 10.1001/ jama.2016.0287

2. Pang T, Fung T, Samra J, Hugh T, Smith R. Pyogenic liver abscess: An audit of 10 years' experience. World Journal of Gastroenterology. 2011;17(12):1622. DOI: 10.3748/wjg.v17.i12.1622

3. Mavilia M, Molina M, Wu G. The Evolving Nature of Hepatic Abscess: A Review. Journal of Clinical and Translational Hepatology. 2016;4(2). DOI: 10.14218/JCTH.2016.00004

4. Cortellaro F, Ferrari L, Molteni F, Aseni P, Velati M, Guarnieri L et al. Accuracy of point of care ultrasound to identify the source of infection in septic patients: a prospective study. Internal and Emergency Medicine. 2016;12(3):371-378. DOI: 10.1007/s11739-016-1470-2

5. Contou D, Roux D, Jochmans S, Coudroy R, Guérot E, Grimaldi D et al. Septic shock with no diagnosis at 24 hours: a pragmatic multicenter prospective cohort study. Critical Care. 2016;20(1). DOI: 10.1186/ s13054-016-1537-5 